\title{
Minimal Explanation of Flavor Anomalies: $B$-Meson Decays, Muon Magnetic Moment, and the Cabibbo Angle
}

\author{
David Marzocca* and Sokratis Trifinopoulos $\odot^{\dagger}$ \\ INFN, Sezione di Trieste, SISSA, Via Bonomea 265, 34136 Trieste, Italy
}

(Received 23 April 2021; accepted 22 June 2021; published 4 August 2021)

\begin{abstract}
Significant deviations from the standard model are observed in semileptonic charged and neutral-current $B$ decays, the muon magnetic moment, and the extraction of the Cabibbo angle. We propose that these deviations point towards a coherent pattern of new physics effects induced by two scalar mediators, a leptoquark $S_{1}$ and a charged singlet $\phi^{+}$. While $S_{1}$ can provide solutions to charged-current $B$ decays and the muon magnetic moment, and $\phi^{+}$can accommodate the Cabibbo-angle anomaly independently, their oneloop level synergy can also address neutral-current $B$ decays. This framework provides the most minimal explanation to the above-mentioned anomalies, while being consistent with all other phenomenological constraints.
\end{abstract}

DOI: 10.1103/PhysRevLett.127.061803

Introduction.-The standard model (SM) of particle physics provides an exquisite description of the interactions between fundamental particles in a very broad spectrum of energies. There are, however, experimental and theoretical reasons to expect departures from the SM due to some new physics (NP) sector. Intriguingly, since a few years certain low-energy flavor measurements pursued at the LHC and several other experiments started exhibiting a number of deviations from SM predictions, which have been growing in significance with the addition of more data.

First, there are hints of lepton flavor universality (LFU) violation in semileptonic $B$-meson decays: (i) $b \rightarrow c \tau \nu$. An enhancement of the charged-current transition in $\tau$ vs light leptons [1-5] with respect to the SM prediction [6-8], as encoded by the ratios

$$
R_{D^{(*)}}=\frac{\mathcal{B}\left(B \rightarrow D^{(*)} \tau \bar{\nu}\right)}{\mathcal{B}\left(B \rightarrow D^{(*)} \ell \bar{\nu}\right)}
$$

is observed at approximately $3 \sigma$. (ii) $b \rightarrow s \ell \ell$. A deficit of the neutral-current transition in muons vs electrons [9-13] manifests in the ratios

$$
R_{K^{(*)}}=\frac{\mathcal{B}\left(B \rightarrow K^{(*)} \mu \bar{\mu}\right)}{\mathcal{B}\left(B \rightarrow K^{(*)} e \bar{e}\right)}
$$

that is predicted to be equal to 1 with high accuracy in the SM [14]. Remarkably, the update on $R_{K}$ presented recently

Published by the American Physical Society under the terms of the Creative Commons Attribution 4.0 International license. Further distribution of this work must maintain attribution to the author(s) and the published article's title, journal citation, and DOI. Funded by SCOAP ${ }^{3}$. by the LHCb Collaboration [13], confirmed the trend observed before and increased the significance of the deviation. Including also an observed deviation in $\mathcal{B}\left(B_{s}^{0} \rightarrow \mu^{+} \mu^{-}\right)$[15-18], that can be precisely predicted in the SM, the combined significance of the deviation reaches $4.7 \sigma$ [19-21]. Furthermore, taking into account also less theoretically clean observables, e.g., differential angular distributions of the $B \rightarrow K^{*} \mu^{+} \mu^{-}$decay, as well as several branching ratios of $b \rightarrow s \mu \mu$ processes [22-24], the overall significance of the deviations in this channel is raised to even above $6 \sigma$, depending on the specific SM prediction employed [19-21,25].

The other two precision measurements featuring anomalous results are (i) $(g-2)_{\mu}$. The long-standing deviation from the SM prediction in the anomalous magnetic moment of the muon $a_{\mu}=(g-2)_{\mu} / 2$ recorded by the BNL experiment [26] has recently been updated by FNAL [27], confirming the previous trend and increasing the significance of the deviation from the SM prediction [28] to an overall $4.2 \sigma$ level [29]. (ii) Cabibbo-angle anomaly (CAA). Discrepancies between the different determinations of the Cabibbo angle were reported recently. In particular, the values of $V_{u s}$ extracted from $K \rightarrow \pi \ell \nu$ decays, the ratio $\mathcal{B}(K \rightarrow \mu \nu) / \mathcal{B}(\pi \rightarrow \mu \nu)$ and CKM unitarity using the value of $V_{u d}$ estimated by superallowed nuclear $\beta$ decays. The tension amounts to $3.6 \sigma$ or $5.1 \sigma[31,32]$ depending on the input from the nuclear $\beta$ decays (i.e., Ref. [33] or Ref. [34]).

In this Letter, we present the minimal ultraviolet (UV) complete NP framework that can provide a combined explanation to the above-mentioned anomalies while being consistent with all other phenomenological constraints. The relevant particle content consists of the $S_{1}$ scalar leptoquark (LQ) and the singly charged scalar $\phi^{+}$, with quantum numbers under $\left(\mathrm{SU}(3)_{c}, \mathrm{SU}(2)_{L}\right)_{\mathrm{U}(1)_{Y}}$ : 


$$
S_{1} \sim(\overline{\mathbf{3}}, \mathbf{1})_{1 / 3}, \quad \phi^{+} \sim(\mathbf{1}, \mathbf{1})_{1} .
$$

The $S_{1}$ LQ has been considered as a mediator for a simultaneous explanation of $R_{D^{(*)}}$, at tree level, and $(g-2)_{\mu}$, at one-loop [35-40]. Additionally, the scalar $\phi^{+}$modifies the tree-level decay of a charged lepton into a lighter one and a neutrino pair, which in turns translates into a shift of $V_{u d}$ necessary to explain the CAA [41-44]. While $S_{1}$ alone cannot explain completely the neutralcurrent anomalies $b \rightarrow s \ell \ell$ via its one-loop contributions $[36,39,45-47]$, we show that the inclusion of an additional box diagram involving both $S_{1}$ and $\phi^{+}$can achieve a very good fit of the data. To this end, we stress that the inclusion of the purely leptonic interactions of $\phi^{+}$, that complement the LQ ones in the full resolution of the $B$-physics anomalies, is fully compatible with the hints towards LFU violation in $\tau$ decays.

We notice that the present model is the most economical. This is due to the fact that none of the proposed one- (or two-particle) solutions can address more than two (or three) out of the four flavor anomalies simultaneously. For instance, the vector LQ models [46,48-54] cannot account either for $(g-2)_{\mu}$ or CAA, and at least two new particles would be necessary in order to improve the combined fit, while the scalar LQ singlet plus triplet solution [37-40] can explain three out of four anomalies without addressing the purely leptonic CAA.

In the following we present the model and perform a global analysis of the anomalous observables and all the relevant constraints, evaluating the improvement over the SM. Finally, we briefly discuss the implications for future experiments.

Model.-The SM Lagrangian is augmented by the following Yukawa-type terms [55]

$\mathcal{L}_{S 1+\phi}=\frac{1}{2} \lambda_{\alpha \beta} \overline{\ell_{\alpha}^{c}} \epsilon \ell_{\beta} \phi^{+}+\lambda_{i \alpha}^{1 L} \overline{q_{i}^{c}} \epsilon \ell_{\alpha} S_{1}+\lambda_{i \alpha}^{1 R} \overline{u_{i}^{c}} e_{\alpha} S_{1}+$ H.c.,

where $\epsilon=i \sigma_{2}$ and we adopt Latin and Greek letters for quark and lepton flavor indices, respectively. The weakdoublets quarks $q_{i}$ and leptons $\ell_{\alpha}$ are in the down-quark and charged-lepton mass eigenstate bases. Note that $\mathrm{SU}(2)_{L}$ invariance enforces antisymmetry of the $\phi^{+}$ couplings to leptons: $\lambda_{\alpha \beta}=-\lambda_{\beta \alpha}$.

It is worth mentioning that the LQ $S_{1}$ and $\phi^{+}$share the same quantum numbers with those of a right-handed sbottom and stau, which might hint of a possible embedding in a SUSY framework. The $\lambda^{1 L}$ and $\lambda$ couplings would then correspond to the $\lambda^{\prime}$ and $\lambda$ ones of the R-parity violating (RPV) superpotential [56] (see also Refs. [57-59]), respectively, while $\lambda^{1 R}$ could potentially originate from nonholomorphic RPV terms [60].
Regarding the couplings employed in the analysis, we do not consider $\lambda^{1 L(R)}$ couplings to the first generation quarks and leptons, as well as $\lambda_{s \mu}^{1 L}$ and $\lambda_{t \tau}^{1 R}$, which are not needed for the explanation of the anomalies. Moreover, we set $\lambda_{e \tau} \approx 0$ in order to satisfy the very strict constraints from the lepton flavor violating (LFV) decay $\mu \rightarrow e \gamma$ [42]. We assume NP couplings to be real, for simplicity.

Observables.-In this section, we present the dominant contributions due to $S_{1}$ and $\phi^{+}$to the anomalous observables. We obtain the $S_{1}$ contributions using the results of Refs. [39,61], to which we refer for more details. In the numerical analysis the complete expressions are employed.

A tree-level $S_{1}$ exchange is invoked in order to explain $b \rightarrow c \tau \nu$ anomalies [see Fig. 1(a)]. The approximate numerical expressions for the $R_{D^{(*)}}$ ratios relevant for the parameter region of interest are

$$
\begin{aligned}
& R_{D} \approx 0.299-0.235 \frac{\lambda_{b \tau}^{1 L} \lambda_{c \tau}^{1 R}}{m_{1}^{2}}\left(1+0.05 \log m_{1}^{2}\right), \\
& R_{D^{*}} \approx 0.258-0.088 \frac{\lambda_{b \tau}^{1 L} \lambda_{c \tau}^{1 R}}{m_{1}^{2}}\left(1+0.02 \log m_{1}^{2}\right),
\end{aligned}
$$

where $m_{1} \equiv M_{1} / \mathrm{TeV}$. Note that quadratic terms and purely left-handed contributions are subleading in our setup. The logarithm becomes important for large masses and enhances the effect in $R_{D}$ compared to $R_{D^{*}}$.

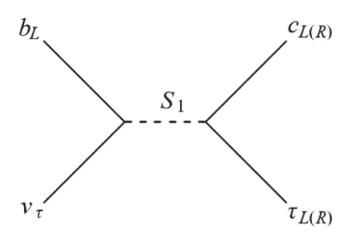

(a)

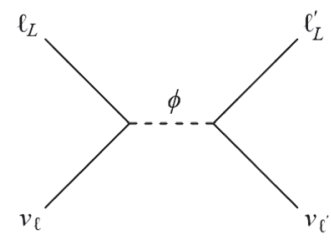

(c)

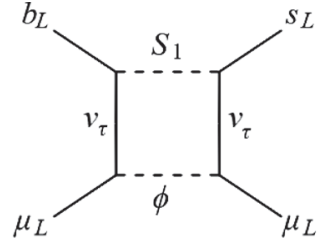

(b)

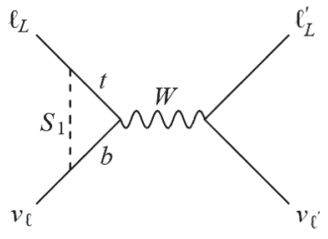

(d)

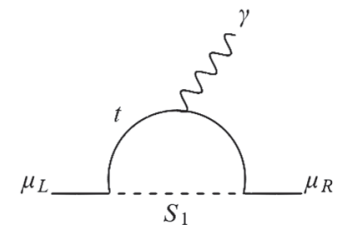

(e)

FIG. 1. The diagrams that generate the dominant contributions to the flavor anomalies. 
The observables related to the $b \rightarrow s \ell \ell$ anomalies receive contributions generated from the Wilson coefficients (WCs) of the operators $\mathcal{O}_{L L(L R)}^{b s \mu \mu}=\left(\bar{s} \gamma^{\alpha} P_{L} b\right)\left(\bar{\mu} \gamma_{\alpha} P_{L(R)} \mu\right)$. They are given by (see also Ref. [35])

$$
\begin{gathered}
C_{L L} \approx-\lambda_{b \tau}^{1 L} \lambda_{s \tau}^{1 L *}\left(\frac{\left|\lambda_{b \mu}^{1 L}\right|^{2}}{64 \pi^{2} M_{1}^{2}}+\frac{\left|\lambda_{\mu \tau}\right|^{2} \log M_{\phi}^{2} / M_{1}^{2}}{64 \pi^{2}\left(M_{\phi}^{2}-M_{1}^{2}\right)}\right), \\
C_{L R} \approx-\frac{\left|\lambda_{c \mu}^{1 R}\right|^{2} \lambda_{b \tau}^{1 L} \lambda_{s \tau}^{1 L *}}{64 \pi^{2} M_{1}^{2}} .
\end{gathered}
$$

The second term in Eq. (7) corresponds to the diagram in Fig. 1(b) and yields the leading contribution in this scenario. Eventually, the results of the global fit are expressed in terms of the low-energy WCs in the standard notation $\Delta C_{9,10}^{\mu}=$ $\left(C_{L R} \pm C_{L L}\right) /\left(2 \mathcal{N}_{s b}\right)$, where $\mathcal{N}_{s b}=G_{F} \alpha V_{t b} V_{t s}^{*} / \sqrt{2} \pi$.

The leading $S_{1}$ contribution to the anomalous muon magnetic moment arises via a triangle diagram [see Fig. 1(e)] and is given by

$$
\Delta a_{\mu} \approx \frac{m_{\mu} m_{t} \lambda_{b \mu}^{1 L} \lambda_{t \mu}^{1 R}}{4 \pi^{2} M_{1}^{2}}\left(\log M_{1}^{2} / m_{t}^{2}-\frac{7}{4}\right) .
$$

The presence of $\phi^{+}$at tree level [see Fig. 1(c)] and $S_{1}$ at one loop [see Fig. 1(d)] implies the following NP effects in the charged-current muon decay:

$\delta(\mu \rightarrow e \nu \nu) \approx \frac{v^{2}\left|\lambda_{e \mu}\right|^{2}}{4 M_{\phi}^{2}}+\frac{3 m_{t}^{2}\left|\lambda_{b \mu}^{1 L}\right|^{2}}{32 \pi^{2} M_{1}^{2}}\left(\frac{1}{2}-\log \frac{M_{1}^{2}}{m_{t}^{2}}\right)$,

where $\delta\left(\ell \rightarrow \ell^{\prime} \nu \nu\right) \equiv \mathcal{A}\left(\ell \rightarrow \ell^{\prime} \nu \nu\right)_{\mathrm{NP}} / \mathcal{A}\left(\ell \rightarrow \ell^{\prime} \nu \nu\right)_{\mathrm{SM}}$.

As investigated in Refs. [31,42], one can alleviate the tension between the value of $V_{u s}$ computed from kaon decays [62], $V_{u s}^{\mathrm{CKM}}=0.2243(5)$ and the one computed via CKM unitarity from $V_{u d}^{\beta}$ as extracted from nuclear beta decays [64], i.e., $V_{u s}^{\beta}=0.2280(6)$, by introducing a constructive interference in $\mu \rightarrow e \nu \nu$. In particular, one obtains

$$
\begin{aligned}
V_{u s}^{\beta} & \equiv \sqrt{1-\left(V_{u d}^{\beta}\right)^{2}-\left|V_{u b}\right|^{2}} \\
& \simeq V_{u s}^{\mathrm{CKM}}\left[1-\left(\frac{V_{u d}^{\mathrm{CKM}}}{V_{u s}^{\mathrm{CKM}}}\right)^{2} \delta(\mu \rightarrow e \nu \nu)\right],
\end{aligned}
$$

where $V_{u d}^{\mathrm{CKM}}=0.97420(21)$ and $\left|V_{u b}\right|^{2} \approx 10^{-5}$ [65] is negligible. Eventually, a global fit including the standard EW observables yields the value of $\delta(\mu \rightarrow e \nu \nu)$ indicated in Table I (see Refs. [31,44] for details).

Phenomenology.-Global analysis.-With all the observables listed in Table I and the expressions for the $S_{1}$ and $\phi^{+}$contributions given here and in the Supplemental Material [74] (which includes the Refs. [39,42,66]) [75], we build a global likelihood $\chi^{2}=-2 \log \mathcal{L}$. We find the best-fit point by minimizing the $\chi^{2}$ function and compare it to the value obtained in the SM.

This analysis prefers large values for the scalar masses $M_{1}$ and $M_{\phi}$. This can be understood by the fact that the
TABLE I. Experimental values for the observables used in the numerical analysis. In the case of $R_{D^{(*)}}, \Delta C_{9,10}^{\mu}$, and $\tau$ LFU the relevant correlations are taken into account.

\begin{tabular}{lc}
\hline \hline Observable & Experimental value \\
\hline$R_{D}$ & $0.34 \pm 0.029[66]$ \\
$R_{D^{*}}$ & $0.295 \pm 0.013[66]$ \\
$\Delta C_{9}^{\mu}$ & $-0.675 \pm 0.16[20]$ \\
$\Delta C_{10}^{\mu}$ & $0.244 \pm 0.13[20]$ \\
$\Delta a_{\mu}$ & $(2.51 \pm 0.59) \times 10^{-9}[27,28]$ \\
$\delta(\mu \rightarrow e \nu \nu)$ & $(6.5 \pm 1.5) \times 10^{-4}[42]$ \\
$R_{D}^{\mu / e}$ & $0.978 \pm 0.035[67,68]$ \\
$\mathcal{B}\left(B_{c} \rightarrow \tau \nu\right)$ & $<0.1[69]$ \\
$R_{K^{(*)}}^{\nu}$ & $<2.7[70]$ \\
$C_{B_{s}}^{1}$ & $<2.01 \times 10^{-5} \mathrm{TeV}^{-2}[71]$ \\
$\left|\operatorname{Re}\left(C_{D}^{1}\right)\right|$ & $<3.57 \times 10^{-7} \mathrm{TeV}^{-2}[71]$ \\
$\left|\operatorname{Im}\left(C_{D}^{1}\right)\right|$ & $<2.23 \times 10^{-8} \mathrm{TeV}^{-2}[71]$ \\
$g_{\tau} / g_{e}$ & $1.0058 \pm 0.0030[66]$ \\
$g_{\tau} / g_{\mu}$ & $1.0022 \pm 0.0030[66]$ \\
$g_{\mu} / g_{e}$ & $1.0036 \pm 0.0028[66]$ \\
$\delta g_{\tau_{L}}^{Z}$ & $(-0.11 \pm 0.61) \times 10^{-3}[72]$ \\
$\delta g_{\tau_{R}}^{Z}$ & $(0.66 \pm 0.65) \times 10^{-3}[72]$ \\
$\delta g_{\mu L}^{Z}$ & $(0.3 \pm 1.1) \times 10^{-3}[72]$ \\
$\delta g_{\mu R}^{Z}$ & $(0.2 \pm 1.3) \times 10^{-3}[72]$ \\
$\mathcal{B}(\tau \rightarrow \mu \gamma)$ & $<4.4 \times 10^{-8}[73]$ \\
$\mathcal{B}(\tau \rightarrow 3 \mu)$ & $<2.1 \times 10^{-8}[73]$ \\
\hline \hline
\end{tabular}

contributions to $b \rightarrow s \mu \mu$ scale as $\lambda^{4} / M^{2}$, while most constraints scale as $\lambda^{2} / M^{2}$, except for $B_{s}$ mixing, which scale with $\lambda^{4} / M^{2}$. Larger masses, and couplings, allow thus to better fit the neutral-current $B$ anomalies and remain compatibile with the other constraints. On the other hand, in order to avoid too large couplings that would put the perturbativity of the model into question, the masses cannot be too large.

Fixing $M_{1}=M_{\phi}=5.5 \mathrm{TeV}$ (we chose equal masses only for simplicity), we find the following best-fit point:

$\lambda_{e \mu}=1.35, \quad \lambda_{\mu \tau}=3.17$,

$\lambda_{b \tau}^{1 L}=1.46, \quad \lambda_{s \tau}^{1 L}=-0.54, \quad \lambda_{b \mu}^{1 L}=2.07$,

$\lambda_{c \tau}^{1 R}=-3.28, \quad \lambda_{t \mu}^{1 R}=0.01, \quad \lambda_{c \mu}^{1 R}=2.35$,

for which $\chi_{\mathrm{SM}}^{2}-\chi_{\text {best-fit }}^{2}=82$ (with $\chi_{\mathrm{SM}}^{2}=110$ for 34 observables included in the fit), which constitutes a major improvement from the SM. The coupling $\lambda_{c \mu}^{1 R}$ is required to cancel an otherwise excessive contribution to $\tau \rightarrow \mu \gamma$. The required cancellation in the amplitude (see Supplemental Material [74]) is approximately of one part in three.

Regarding the RG evolution of the couplings, we find that $\lambda_{c \tau}^{1 R}$ reaches a Landau pole at a scale of about $20 \mathrm{TeV}$. A UV completion for this model should therefore appear before that scale to address this issue, a possibility being a composite scenario similar to the one studied in Ref. [76]. 

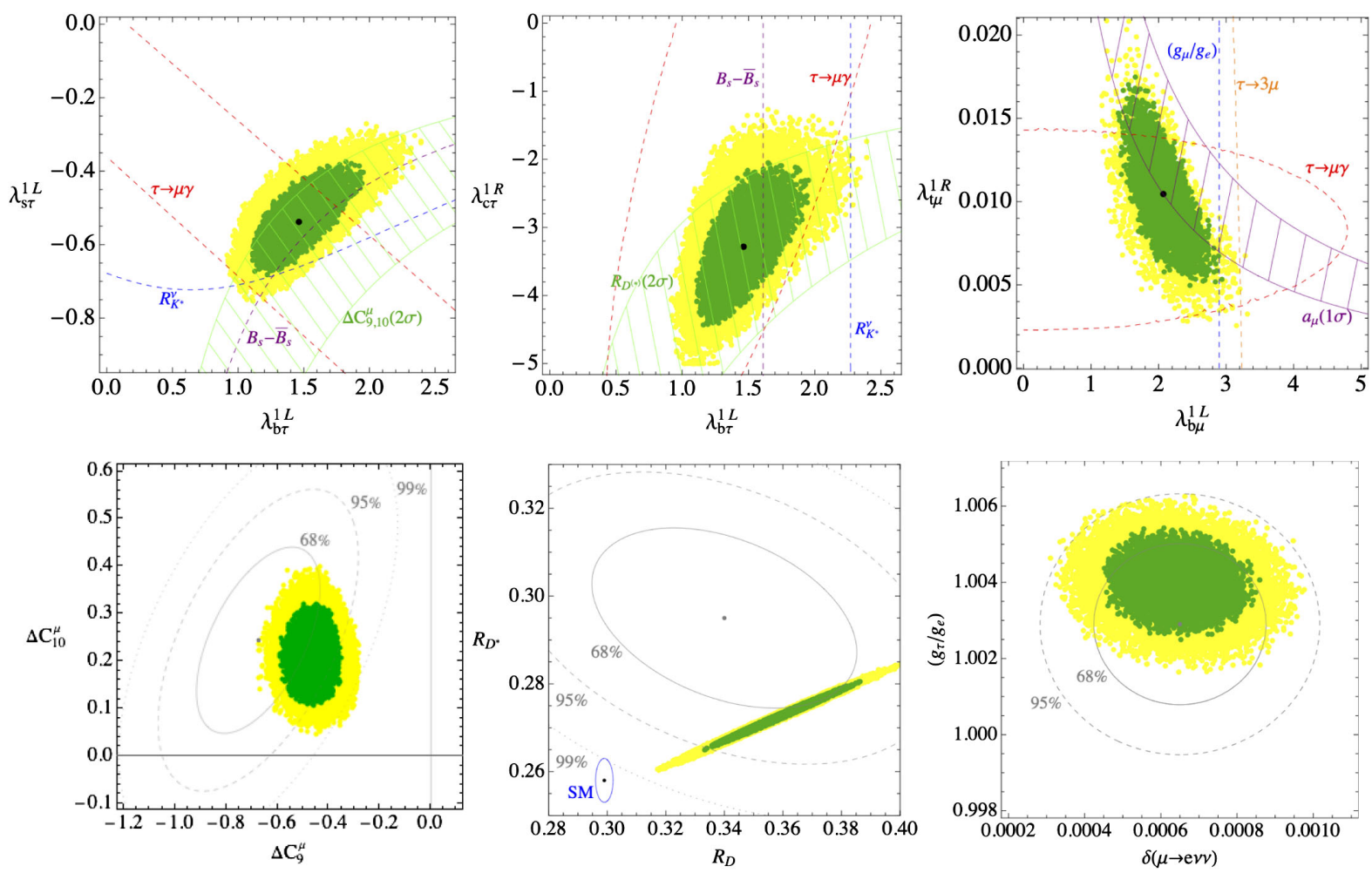

FIG. 2. Results of the parameter scan of the model's parameters, with $M_{1}=M_{\phi}=5.5 \mathrm{TeV}$. The green (yellow) points are within $1 \sigma$ $(2 \sigma)$ of the best-fit point, shown in black. The upper row shows the preferred region for some of the couplings and the single-observable 95\% C.L. constraints. The bottom row shows how this preferred region maps in the plane of pairs of observables of interest.

We also checked that all the couplings we set to be very small remain stable even after RG evolution.

To study the preferred region in parameter space we perform a numerical scan via a Markov-chain Monte Carlo algorithm that we use to select points with $\Delta \chi^{2}=\chi^{2}-\chi_{\text {best-fit }}^{2}$ corresponding to $68 \%$ and $95 \%$ confidence level (CL) regions. The results of this scan are shown in Fig. 2. In the top row we show the preferred regions for pairs of couplings as well as the relevant single-observable constraints in each plane [77]. In the bottom row we show how these preferred regions map into pairs of the observables showing a discrepancy with the SM (the effect in $\Delta a_{\mu}$ can be seen in the top-right plot comparing with the purple-meshed region representing the experimentally preferred value at $1 \sigma$ ).

We observe that the model is able to address at the $1 \sigma$ level all the four deviations from the SM presented in the introduction. As a by-product, a small tension present in LFU tests in $\tau$ decays, $\left(g_{\tau} / g_{e}\right)$, is also addressed in this framework.

Future prospects.-Both $B_{s}$ mixing and $B \rightarrow K^{(*)} \nu \nu$ are sensitive to the $S_{1}$ couplings contributing to $R_{K^{(*)}}$ and $R_{D^{(*)}}$, and the preferred region by the model is close to the present exclusion limits, as shown in Fig. 3 for $R_{K^{*}}^{\nu}$. A deviation from the SM could thus reveal itself in future updates of this observable by the Belle-II experiment [78].

Via a one-loop box diagram with both $S_{1}$ and $\phi^{+}$, similar to Fig. 1(b), a contribution to the LFV process $b \rightarrow s \mu e$ is induced. The preferred values in our model for $\mathcal{B}(B \rightarrow K \mu e)$ are shown in Fig. 3, while $\mathcal{B}\left(B \rightarrow K^{*} \mu e\right) \approx$ $2.1 \mathcal{B}(B \rightarrow K \mu e)$ and $\mathcal{B}\left(B_{s} \rightarrow \mu e\right) \sim \mathcal{O}\left(10^{-12}\right)$. On the other hand, due to the specific structure of the couplings, in this model we do not predict sizable effects in $b \rightarrow s \tau \tau$ and $b \rightarrow s \tau \mu$ processes.

As shown in Fig. 2 (bottom-right) we also expect permille effects in LFU tests in $\tau$ decays, which is in the range

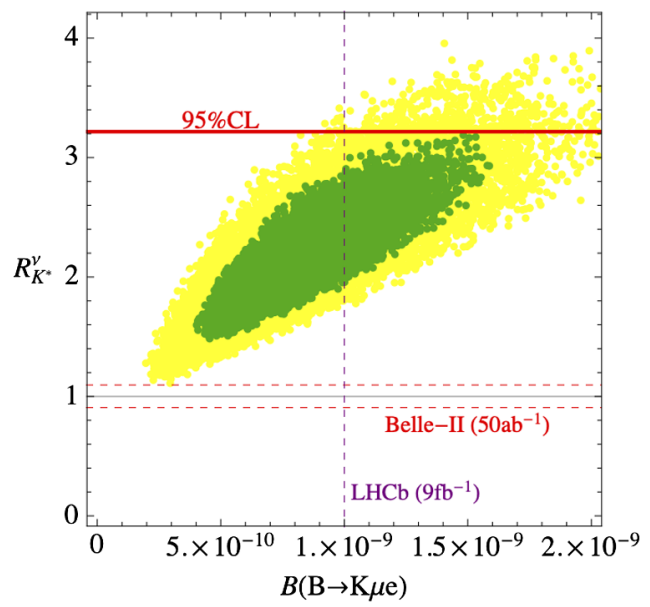

FIG. 3. Here the preferred values of $\mathcal{B}(B \rightarrow K \mu e)$ and $R_{K^{*}}^{\nu}$ are shown, together with the present $95 \%$ C.L. limit (red line) and the future prospects expected by LHCb [79] and Belle II [78]. 
of future sensitivity by Belle-II [78]. The model predicts also effects in LFV $\tau$ decays. The $S_{1}$ LQ generates $\tau \rightarrow \mu \gamma$, $\tau \rightarrow 3 \mu$, and $\tau \rightarrow \mu e e$ with rates close to the present bounds (of the order of $\sim 10^{-8}$ ). The scalar $\phi^{+}$, instead, mediates $\mathcal{B}(\tau \rightarrow e \mu \mu) \sim 10^{-9}, \mathcal{B}(\tau \rightarrow 3 e) \sim 10^{-10}$, and $\mathcal{B}(\tau \rightarrow e \gamma) \sim$ $10^{-11}$ [42]. Also for these channels Belle-II and LHCb are expected to improve substantially on the present constraints by at least 1 order of magnitude [78,79].

Finally, while the large masses preferred by the fit are beyond the reach of direct searches at LHC, effects in highenergy tails of Drell-Yan due to $S_{1}$ are possible. At FCC-hh the leptoquark could be produced on-shell and a muon collider would be the ideal machine to study also the scalar $\phi^{+}$.

Conclusions. - In this Letter we propose a new physics model addressing the most significant deviations from the SM observed in flavor physics, while being at the same time consistent with all the relevant phenomenological constraints. The model is the first one that establishes a connection between all four classes of flavor anomalies under the same LFU violating interpretation. Furthermore, since it comprises of only two weak-singlet scalars: the leptoquark $S_{1}$ and the colorless $\phi^{+}$, it is also the most minimal solution to be proposed in the literature for a combined resolution of them.

In the foreseeable future, the $\mathrm{LHCb}$ and Belle-II experiments will clarify the nature of the present anomalies in $B$ decays, while the Fermilab $(g-2)_{\mu}$ experiment has already been collecting a large amount of additional data that will allow us to further reduce the experimental uncertainty. In order to settle the CKM unitarity puzzle, experimental developments are expected in the existing precision observables used for the determination of the Cabibbo angle [80] as well as further observables such as hadronic $\tau$ decays [81], the pion $\beta$ decay [82] and the neutron lifetime [33] that can provide complementary tests in the future.

If any one of these signals will be further confirmed by future data it would imply a revolution in our understanding of fundamental interactions. However, it is only by the combination of several deviations in different observables that we might be able to pinpoint the precise nature of the underlying new physics.

The authors acknowledge support by MIUR Grant No. PRIN 2017L5W2PT. D. M. is also partially supported by the INFN grant SESAMO and by the European Research Council (ERC) under the European Unions Horizon 2020 research and innovation programme, Grant Agreement No. 833280 (FLAY).

Note added in the proof.-A study with an explanation of $(g-2)_{\mu}$ and $b \rightarrow s \ell \ell$ anomalies that includes a successful dark matter candidate is also being published in this journal [83]. *david.marzocca@ts.infn.it

† sokratis.trifinopoulos@ts.infn.it

[1] J. P. Lees et al. (BABAR Collaboration), Phys. Rev. D 88, 072012 (2013).

[2] S. Hirose et al. (Belle Collaboration), Phys. Rev. Lett. 118, 211801 (2017).

[3] R. Aaij et al. (LHCb Collaboration), Phys. Rev. Lett. 115, 111803 (2015); 115, 159901(E) (2015).

[4] R. Aaij et al. (LHCb Collaboration), Phys. Rev. D 97, 072013 (2018).

[5] A. Abdesselam et al. (Belle Collaboration), arXiv:1904 .08794 .

[6] F. U. Bernlochner, Z. Ligeti, M. Papucci, and D. J. Robinson, Phys. Rev. D 95, 115008 (2017); 97, 059902 (E) (2018).

[7] D. Bigi, P. Gambino, and S. Schacht, J. High Energy Phys. 11 (2017) 061.

[8] S. Jaiswal, S. Nandi, and S. K. Patra, J. High Energy Phys. 12 (2017) 060.

[9] R. Aaij et al. (LHCb Collaboration), J. High Energy Phys. 08 (2017) 055.

[10] R. Aaij et al. (LHCb Collaboration), Phys. Rev. Lett. 122, 191801 (2019).

[11] A. Abdesselam et al. (Belle Collaboration), Phys. Rev. Lett. 126, 161801 (2021).

[12] S. Choudhury et al. (BELLE Collaboration), J. High Energy Phys. 03 (2021) 105.

[13] R. Aaij et al. (LHCb Collaboration), arXiv:2103.11769.

[14] M. Bordone, G. Isidori, and A. Pattori, Eur. Phys. J. C 76, 440 (2016).

[15] R. Aaij et al. (LHCb Collaboration), J. High Energy Phys. 09 (2015) 179.

[16] R. Aaij et al. (LHCb Collaboration), Phys. Rev. Lett. 118, 191801 (2017).

[17] M. Aaboud et al. (ATLAS Collaboration), J. High Energy Phys. 04 (2019) 098.

[18] A. M. Sirunyan et al. (CMS Collaboration), J. High Energy Phys. 04 (2020) 188.

[19] L. S. Geng, B. Grinstein, S. Jäger, S. Y. Li, J. Martin Camalich, and R.X. Shi, arXiv:2103.12738.

[20] W. Altmannshofer and P. Stangl, arXiv:2103.13370.

[21] M. Algueró, B. Capdevila, A. Crivellin, S. Descotes-Genon, P. Masjuan, J. Matias, M. Novoa Brunet, and J. Virto, Eur. Phys. J. C 79, 714 (2019).

[22] R. Aaij et al. (LHCb Collaboration), Phys. Rev. Lett. 111, 191801 (2013).

[23] R. Aaij et al. (LHCb Collaboration), J. High Energy Phys. 02 (2016) 104.

[24] R. Aaij et al. (LHCb Collaboration), Phys. Rev. Lett. 125, 011802 (2020).

[25] M. Ciuchini, A. M. Coutinho, M. Fedele, E. Franco, A. Paul, L. Silvestrini, and M. Valli, Eur. Phys. J. C 79, 719 (2019).

[26] G. W. Bennett et al. (Muon g-2 Collaboration), Phys. Rev. D 73, 072003 (2006).

[27] B. Abi et al. (Muon g-2 Collaboration), Phys. Rev. Lett. 126, 141801 (2021)

[28] T. Aoyama, N. Asmussen, M. Benayoun, J. Bijnens, T. Blum, M. Bruno, I. Caprini, C. M. Carloni Calame, M. Cè, G. Colangelo et al., Phys. Rep. 887, 1 (2020). 
[29] See, however, Ref. [30], that claims a much reduced discrepancy between SM and measurement, as well as the corresponding discussion in Ref. [28].

[30] S. Borsanyi, Z. Fodor, J. N. Guenther, C. Hoelbling, S. D. Katz, L. Lellouch, T. Lippert, K. Miura, L. Parato, K. K. Szabo et al., Nature (London) 593, 51 (2021).

[31] B. Belfatto, R. Beradze, and Z. Berezhiani, Eur. Phys. J. C 80, 149 (2020).

[32] Y. Grossman, E. Passemar, and S. Schacht, J. High Energy Phys. 07 (2020) 068.

[33] A. Czarnecki, W. J. Marciano, and A. Sirlin, Phys. Rev. D 100, 073008 (2019).

[34] C. Y. Seng, M. Gorchtein, and M. J. Ramsey-Musolf, Phys. Rev. D 100, 013001 (2019).

[35] M. Bauer and M. Neubert, Phys. Rev. Lett. 116, 141802 (2016).

[36] Y. Cai, J. Gargalionis, M. A. Schmidt, and R. R. Volkas, J. High Energy Phys. 10 (2017) 047.

[37] A. Crivellin, D. Müller, and F. Saturnino, J. High Energy Phys. 06 (2020) 020.

[38] S. Saad, Phys. Rev. D 102, 015019 (2020).

[39] V. Gherardi, D. Marzocca, and E. Venturini, J. High Energy Phys. 01 (2021) 138.

[40] H. M. Lee, Phys. Rev. D 104, 015007 (2021).

[41] A. Crivellin, C. A. Manzari, M. Alguero, and J. Matias, Phys. Rev. Lett. 127, 011801 (2021)..

[42] A. Crivellin, F. Kirk, C. A. Manzari, and L. Panizzi, Phys. Rev. D 103, 073002 (2021).

[43] T. Felkl, J. Herrero-Garcia, and M. A. Schmidt, J. High Energy Phys. 05 (2021) 122.

[44] A. Crivellin, M. Hoferichter, and C. A. Manzari, arXiv: 2102.02825.

[45] A. Azatov, D. Barducci, D. Ghosh, D. Marzocca, and L. Ubaldi, J. High Energy Phys. 10 (2018) 092.

[46] A. Angelescu, D. Bečirević, D. A. Faroughy, and O. Sumensari, J. High Energy Phys. 10 (2018) 183.

[47] A. Angelescu, D. Bečirević, D. A. Faroughy, F. Jaffredo, and O. Sumensari, arXiv:2103.12504.

[48] R. Barbieri, G. Isidori, A. Pattori, and F. Senia, Eur. Phys. J. C 76, 67 (2016).

[49] D. Buttazzo, A. Greljo, G. Isidori, and D. Marzocca, J. High Energy Phys. 11 (2017) 044.

[50] L. Di Luzio, A. Greljo, and M. Nardecchia, Phys. Rev. D 96, 115011 (2017).

[51] M. Bordone, C. Cornella, J. Fuentes-Martin, and G. Isidori, Phys. Lett. B 779, 317 (2018).

[52] L. Calibbi, A. Crivellin, and T. Li, Phys. Rev. D 98, 115002 (2018).

[53] M. Blanke and A. Crivellin, Phys. Rev. Lett. 121, 011801 (2018).

[54] C. Cornella, D. A. Faroughy, J. Fuentes-Martín, G. Isidori, and M. Neubert, arXiv:2103.16558.

[55] In principle, there exist also quartic couplings between the scalars themselves and between the scalars and the Higgs boson. They are not relevant for the phenomenological analysis of this work and are thus omitted.

[56] R. Barbier, C. Berat, M. Besancon, M. Chemtob, A. Deandrea, E. Dudas, P. Fayet, S. Lavignac, G. Moreau, E. Perez, and Y. Sirois, Phys. Rep. 420, 1 (2005).

[57] S. Trifinopoulos, Eur. Phys. J. C 78, 803 (2018).
[58] S. Trifinopoulos, Phys. Rev. D 100, 115022 (2019).

[59] W. Altmannshofer, P. S. B. Dev, A. Soni, and Y. Sui, Phys. Rev. D 102, 015031 (2020).

[60] C. Csaki, E. Kuflik, and T. Volansky, Phys. Rev. Lett. 112, 131801 (2014).

[61] V. Gherardi, D. Marzocca, and E. Venturini, J. High Energy Phys. 07 (2020) 225; 01 (2021) 006(E).

[62] This is an average of the value extracted from $K \rightarrow \pi \ell \nu$ decays $V_{u s}^{K \ell 3}=0.22326(58)$ and the ratio $\mathcal{B}(K \rightarrow \mu \nu) /$ $\mathcal{B}(\pi \rightarrow \mu \nu), V_{u s}^{K \mu 2}=0.22534(42)$ [63]. Note that the discrepancy between $V_{u s}^{K \ell 3}$ and $V_{u s}^{K \mu 2}$ cannot be explained by LFU violation.

[63] S. Aoki et al. (Flavour Lattice Averaging Group), Eur. Phys. J. C 80, 113 (2020).

[64] C. Y. Seng, X. Feng, M. Gorchtein, and L. C. Jin, Phys. Rev. D 101, 111301(R) (2020).

[65] M. Tanabashi et al. (Particle Data Group), Phys. Rev. D 98 , 030001 (2018).

[66] Y. S. Amhis et al. (HFLAV Collaboration), Eur. Phys. J. C 81, 226 (2021).

[67] B. Aubert et al. (BABAR Collaboration), Phys. Rev. D 79, 012002 (2009).

[68] R. Glattauer et al. (Belle Collaboration), Phys. Rev. D 93, 032006 (2016).

[69] A. G. Akeroyd and C. H. Chen, Phys. Rev. D 96, 075011 (2017).

[70] J. Grygier et al. (Belle Collaboration), Phys. Rev. D 96, 091101 (2017).

[71] M. Bona et al. (UTfit Collaboration), J. High Energy Phys. 03 (2008) 049.

[72] S. Schael et al. (ALEPH, DELPHI, L3, OPAL, SLD, LEP Electroweak Working Group, SLD Electroweak Group, and SLD Heavy Flavour Group), Phys. Rep. 427, 257 (2006).

[73] C. Patrignani et al. (Particle Data Group), Chin. Phys. C 40, 100001 (2016).

[74] See Supplemental Material at http://link.aps.org/ supplemental/10.1103/PhysRevLett.127.061803 for approximate expressions for the observables listed in Table I.

[75] We define $R_{D}^{\mu / e}=\mathcal{B}(B \rightarrow D \mu \nu) / \mathcal{B}(B \rightarrow D e \nu), \quad R_{K^{(*)}}^{\nu}=$ $\mathcal{B}\left(B \rightarrow K^{(*)} \nu \nu\right) / \mathcal{B}\left(B \rightarrow K^{(*)} \nu \nu\right)_{\mathrm{SM}}, C_{B_{s}(D)}^{1}$ as the coefficients of the corresponding $\Delta F=2$ operator $\left(\bar{q}_{L}^{i} \gamma_{\mu} q^{j}\right)^{2}$, and $g_{\mu} / g_{e}=|[1+\delta(\tau \rightarrow \mu \nu \nu)] /[1+\delta(\tau \rightarrow e \nu \nu)]| \quad$ (and similarly for the others, see Ref. [66]).

[76] D. Marzocca, J. High Energy Phys. 07 (2018) 121.

[77] For brevity we do not show analogous plots for $\lambda_{c \mu}^{1 R}$, which has values in the interval $1.5,3]$, and the $\phi^{+}$couplings, which take values $\lambda_{e \mu} \in[1.1,1.6]$ and $\lambda_{\mu \tau} \in[2.7,3.6]$.

[78] E. Kou et al. (Belle-II Collaboration), Prog. Theor. Exp. Phys. 2019, 123 C01 (2019); 2020, 029201(E) (2020).

[79] R. Aaij et al. (LHCb Collaboration), arXiv:1808.08865.

[80] A. Crivellin and M. Hoferichter, Phys. Rev. Lett. 125, 111801 (2020).

[81] A. Lusiani, EPJ Web Conf. 218, 05002 (2019).

[82] A. Czarnecki, W. J. Marciano, and A. Sirlin, Phys. Rev. D 101, 091301(R) (2020).

[83] G. Arcadi, L. Calibbi, M. Fedele, and F. Mescia, preceding Letter, Phys. Rev. Lett. 127, 061801 (2021). 